\title{
Social and Biological Issues in Infant Survivorship among Danish Cohorts Born between 1982 and 1990
}

\author{
HANS O. HANSEN \\ Associate Professor of Demography \\ Institute of Statistics \\ Copenhagen University \\ Copenhagen, Denmark
}

\begin{abstract}
The purpose of this project is to identify possible differentials in the infant survivorship of the Danish cohorts born between 1982 and 1990. The principal characteristics to be considered are gender and birth weight. Our data consist of official records of live births and infant deaths linked at the individual level. We report some rather detailed measurements of the survivorship impact of sex and birth weight in the framework of logistic regression and loglinear modeling. This paper gives strong support to sex and birth weight as major determinants of infant survivorship. Falling infant mortality is closely associated with increasing expected birth weight over the birth cohorts considered. The present paper should be seen as an appetizer for addressing the more general question of birth weight as an intermediate variable for survivorship impacts of biosocial factors related to the parents and to intrauterine gestation.
\end{abstract}

Keywords: infant mortality; birth weight, differential mortality, Denmark

\section{Introduction}

Sudden infant death or deteriorating child health, eventually leading to death, may be caused by congenital biosocial factors and/or by inoptimal or untimely childcare.

The social and biological factors in gestation and infant survivorship are complexly interrelated. For example, the smoking habits of the mother are known to be conducive to low birth weight. See for example Cederqvist et al. (1984), Tambyraja and Ratnam (1982) and Phelan (1980). Alcoholism and drug abuse are other behavioral factors that may damage the fetus and the child. The age, health and nutrition status of the mother, the age of the father, and the genetic heritage of the parents have an impact on the health and the genetical disposition of the offspring.

From the literature, biological factors such as sex and birth weight are known to be important determinants of infant mortality, more so in the perinatal and neonatal age intervals than later in life. See for example Belsey (1993) and Bouvier and Van der Tak (1976). Low birth weight is frequently associated with an increased mortality above that associated with normal birth weight; boys usually having higher mortality 
than girls. Biological factors, obviously, may also be important contributing elements in the shaping of the social life and the career opportunities of the new-born child.

In this study we investigate selected issues in infant survivorship among the Danish cohorts born between 1982 and 1990. Our data consist of official records of live births and infant deaths linked at the individual level.

Fundamentally, this paper gives strong support to the alleged impacts of sex and birth weight upon infant survivorship, the relationship being particularly simple in the medium range of the birth weight distributions. Is this a trivial finding? We think not. The estimated infant mortality is below one percent in the cohorts under study. Not surprisingly, this adds to the variation of the observed birth weights. The supreme data quality and the sheer size of the data set permits highly accurate assessment, not only in the medium range of the birth weight distributions, but also in their extremes. With specific regard to the tails of the birth weight distribution, we find a looser association between gender and birth weight, on the one hand, and infant survivorship on the other.

Until recent years infant mortality has been somewhat higher in Denmark relative to other Nordic countries. Falling Danish infant mortality is closely associated with systematically increasing estimated expected birth weight (in the range of 1,000 to 4,499 grams) over the birth cohorts considered. This result calls for cross-national comparisons of birth weight distributions. The last-mentioned research theme will not be pursued further here.

The present paper should also be seen as an appetizer for addressing the more general question of birth weight as an intermediate variable for survivorship impact of biosocial factors related to the parents and to intrauterine gestation. An exhaustive treatment of this matter, clearly, is beyond the scope of a short paper.

A brief presentation of the data is given in the next section. Further specification of the problem and some detailed research issues to be derived from it follow in the section dealing with problems. A brief review of the applied statistical models and the statistical analysis are given in the next section. The findings are presented and summarized in the results section. A brief discussion follows in the conclusion.

\section{The data}

The empirical basis for addressing questions of the above-mentioned nature is a very large data set consisting of the birth records of live born Danish children delivered between January 1, 1982 and December 31, 1992, and a much smaller data set consisting of individual birth and death records of children belonging to the first-mentioned data set and dying before their first birthday.

At the present stage of the project we have been forced to exclude children born in the second half of the year 1987 due to an irrecoverable error on one of the diskettes received from the donor of the data (Sundhedsstyrelsen). As for now, we will also omit children born in 1991 and 1992 due to a shift in the reporting practice from the year 1991. We hope to include all omitted birth cohorts and the related survivorship experience at a later stage of research. At present, the resulting analytic datafile includes 475,714 live births and 3,746 deaths.

In Denmark, the registration of births and deaths has been universal and highly accurate for a very long time. The fraction of sampled live births, consequently, is close to 1 .

The analytic datafile permits assessment of individual censored or uncensored lifetimes with great precision. On a retrospective basis the data convey some background information on the parents and some (rather imperfect) information on intrauterine ev- 
olution from gestation to birth. From live birth to death, to censoring at the first birthday, to censoring before 1st January, 1991, the available survivorship data fit into a prospective observational plan. Censoring in terms of external out-migration is not reported in the data.

The state space of the data and the underlying stochastic life models is graphed in Figure 1.

Fig u re 1. State space of the data and the life models.

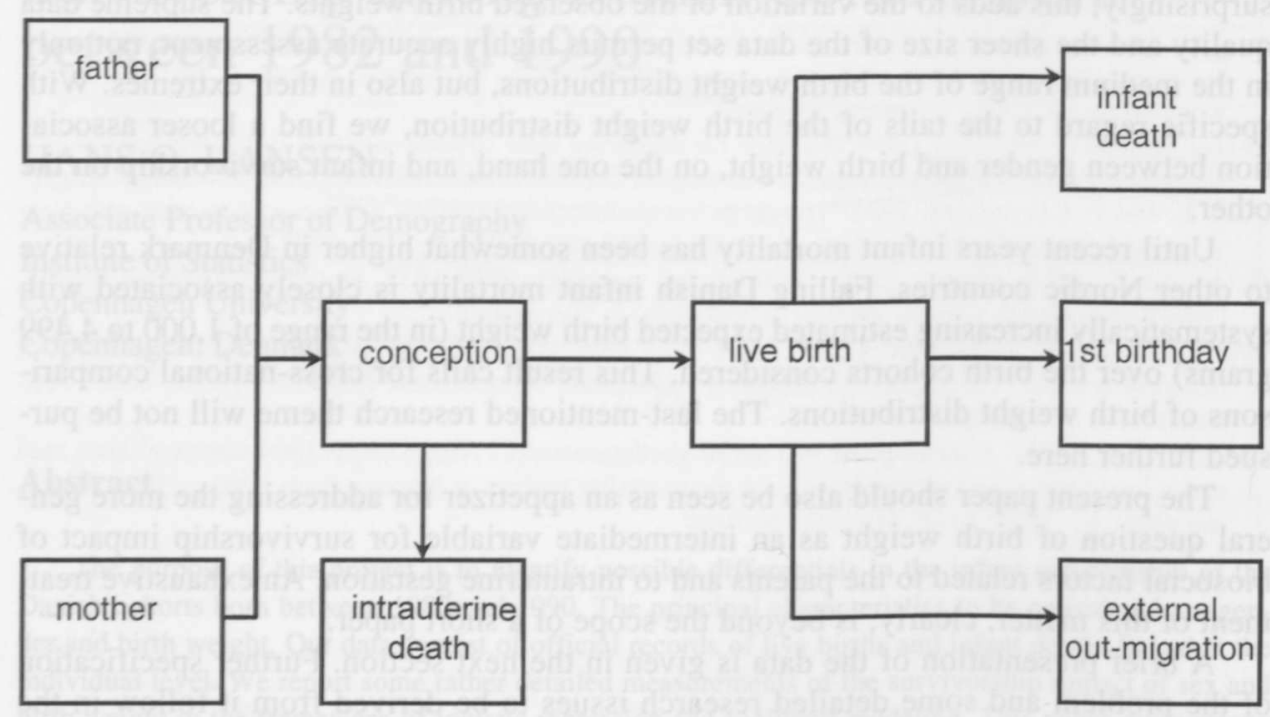

\section{Problems}

What makes birth weight such an important determinant of infant survivorship? First of all its measurability, of course. In medical practice, body length is commonly used as a proxy for intrauterine gestation age. Normally, birth weight would be positively correlated with body length. As birth weight may be easier to determine accurately than body length at birth, birth weight stands out as a very suitable intermediate indicator of gestation age. Low birth weight related to prematurity is known to impair the survivorship prospects of live-born babies. This will also become abundantly evident in the following sections.

Observed weight distributions of live births obviously are functions of observable or latent biosocial factors, commonly of great complexity. The quality and availability of the medical care and obstetrical skills may, to a very large extent, offset adverse biosocial dispositions with the fetus and/or the pregnant women; of consequence for the delivery; and for the subsequent survivorship of live-born babies. The sore issues of the social costs and benefits involved with preserving life before and after birth will not be further considered in this paper.

In what range of a continuos weight scale would the odds of surviving at least until a given age before the first birthday be best? And how is survivorship affected by extreme birth weight, either low or high?

If birth weight is differential with regard to gender, some interaction is to be expected between these covariates with respect to survivorship. 
The question of a measurable relationship between infant survivorship and birth year clearly needs some qualification. Infant mortality is heavily dependent on age. Due to selection impacts of care and medical treatment, transition to "unavoidable" death clearly may be "deferred" or "postponed" to a somewhat higher age, relatively speaking. In other terms, prevention of death is likely to affect the "frailty" or health composition of the survivors at a given age.

To study the time dependency of infant mortality as a function of gender and birth weight, some sub-categorization of the age segment "first year-of-life" will be required.

To ensure comparability and to avoid distracting details at this point, we shall use the established age categorization of infants from the literature; i.e. (in units of days of life) $[0,1[,[1,7],[7,27[$ (the neonatal period), and [28,1st birthday[ (the postneonatal period); the sum of the first two segments referring to the perinatal period.

\section{Model and statistical analysis}

The joint survivorship impact of gender and birth weight are evaluated in the framework of a logistic regression model. Given survivorship experience in terms of a multivariate contingency table, the estimation and the related model control were accomplished with the BMDP procedure LR.

To disentangle possible interactions in the background variables, we use loglinear modeling in terms of the BMDP procedure 4F. This was done to make it easier to understand some of the regression results.

DEMOPACK vers. 1.1 (Hansen 1993) and MINISTAT vers. 1.0 (Hansen 1994) served as efficient and straightforward tools on performing some preliminary and associated demographic and statistical analyses.

\section{Results}

To address the questions raised in the Problems section, we will first examine the univariate distributions of live births by gestation age, birth weight, and body length.

To identify strategic points on a continuous scale for birth weight in regard to the multivariate statistical analyses, we will then focus on the nature of the mutual relationship of birth weight, body length at birth, and cohort.

Using a partitioning based on the identified strategic points in the distribution by birth weight, we will finally report some rather detailed measurements of the survivorship impact of sex and birth weight, partly in terms of estimated probabilities, and partly in terms of regression results.

\section{Analysis of the univariate distributions of live births by birth weight and body length}

The examination of the univariate distributions of live births by birth and body length is based on elementary statistical methodology associated with the normal distribution.

Assume that $\mathbf{X}$ is a normally distributed stochastic variable with mean $\mu$ and standard deviation $\sigma$ i.e.,

$$
\mathrm{H}_{\mathrm{o}}: \mathrm{X} \sim \mathrm{N}(\mu, \sigma)
$$

The working hypothesis $\mathrm{H}_{\mathrm{o}}$ may be controlled by a fractile diagram. The graphical control of the assumed distribution may be supported by a (numerical) goodness- 
of-fit test. The graphical control is based on a linear transformation of the (grouped) stochastic variable $\mathbf{X}$,

$$
\mathrm{U}=(\mathrm{X}-\mu) / \sigma \sim \mathrm{N}(0,1)
$$

In the fractile diagrams, the dependent $\mathrm{u}$-scores are represented by the first $\mathrm{Y}$-axis, while the second $\mathrm{Y}$-axis refers to the empirical distribution $\mathrm{H}(\mathrm{x})$. The diagrams to be presented in the following, consequently, serve the twin purposes of checking the hypothesized normal distribution of the stochastic variable under study and of conveying detailed information on the fractiles $\mathrm{x}_{\mathrm{p}}$ based on the empirical distribution $\mathrm{H}\left(\mathrm{x}_{\mathrm{p}}\right)=\mathrm{est} . \mathrm{P}\left(\mathrm{X} \leq \mathrm{x}_{\mathrm{p}}\right)$. Fractile diagrams of distributions by birth weight are shown in Figure 2 (both sexes) and in Figures A1-A2, Appendix 2 (boys; girls); fractile diagrams of distributions by body length at live birth are displayed in Figure 3 (both sexes) and in Figures A3-A4, Appendix 2 (boys; girls). The ML-estimates of parameters $\mu$ and $\sigma$ are shown in Table 1 . Newborn boys are taller and heavier than newborn girls. Using approximative testing based on the normal distribution, these differentials are found to be statistically significant. Fitted normal densities of distributions by length at birth and birth weight are shown in Figures $4-5$. The graphs refer to all live births. The densities are based on the parameter estimates in Table 1. Similar graphs by sex are shown in Figures A5-A6 (birth weight), Appendix 2, and in Figures A7-A8 (length at live birth), Appendix 2.

There exist a seemingly next-to-perfect linear relationship between the u-scores and the independent $\mathrm{x}$-variable birth weight (/body length at birth) in all six fractile diagrams. Can the result obtained by graphical inspection of the fractile diagrams and the underlying densities be maintained when supplemented with goodness-of-fit testing? The answer is no. Contrary to the graphical approach, the numerical test is highly sensitive to the number of observations. When applied to very large data sets, the power of the applied chi-square test becomes extremely high. The empirical density of birth weight is almost perfectly bell-shaped. The empirical density is somewhat higher than the normal density in the medium range of c. 2,500 grams to c. 4,500 grams; to a large extent this is also true of length at live birth in the medium range of some $47 \mathrm{~cm}$ to some $52 \mathrm{~cm}$. Structural distributional deviations of this nature could perhaps be mediated by introducing background variables like birth year and birth weight. Detailed results of that nature will not be reported here.

T a b le 1. Estimated birth weight and body length at birth, live births by sex 1982 1990 (+) (metric scale).

$\begin{array}{lll}\begin{array}{l}\text { Sex } \\ \text { Estimate } \\ \text { Boys } \\ \text { mean }\end{array} & \begin{array}{l}\text { Birth weight } \\ \text { (grams) }\end{array} & \begin{array}{l}\text { Length at birth } \\ (\mathrm{cm})\end{array} \\ \text { SD } & 3,470.98 & 52.40 \\ \quad \text { \# of cases used } & 592.42 & 2.69 \\ \quad \text { \# of cases omitted } & 243,921 & 240,244 \\ \text { Girls } & 357 & 4,034 \\ \quad \text { mean } & & \\ \text { SD } & 3,351.71 & 51.64 \\ \text { \# of cases used } & 559.08 & 2.55 \\ \quad \text { \# of cases omitted } & 231,115 & 227,941 \\ \text { Both sexes } & 321 & 3,495 \\ \quad \text { mean } & & \\ \text { SD } & 3,412.96 & 52.03 \\ \text { \# of cases used } & 579.51 & 2.65 \\ \text { \# of cases omitted } & 475,036 & 468,185 \\ \text { (+) excling live birth } & 678 & 7,529\end{array}$

(+) excluding live births during the latter half of 1987. 
Fi g u re 2. Graphic control of assumed normal distribution, all birth weights.

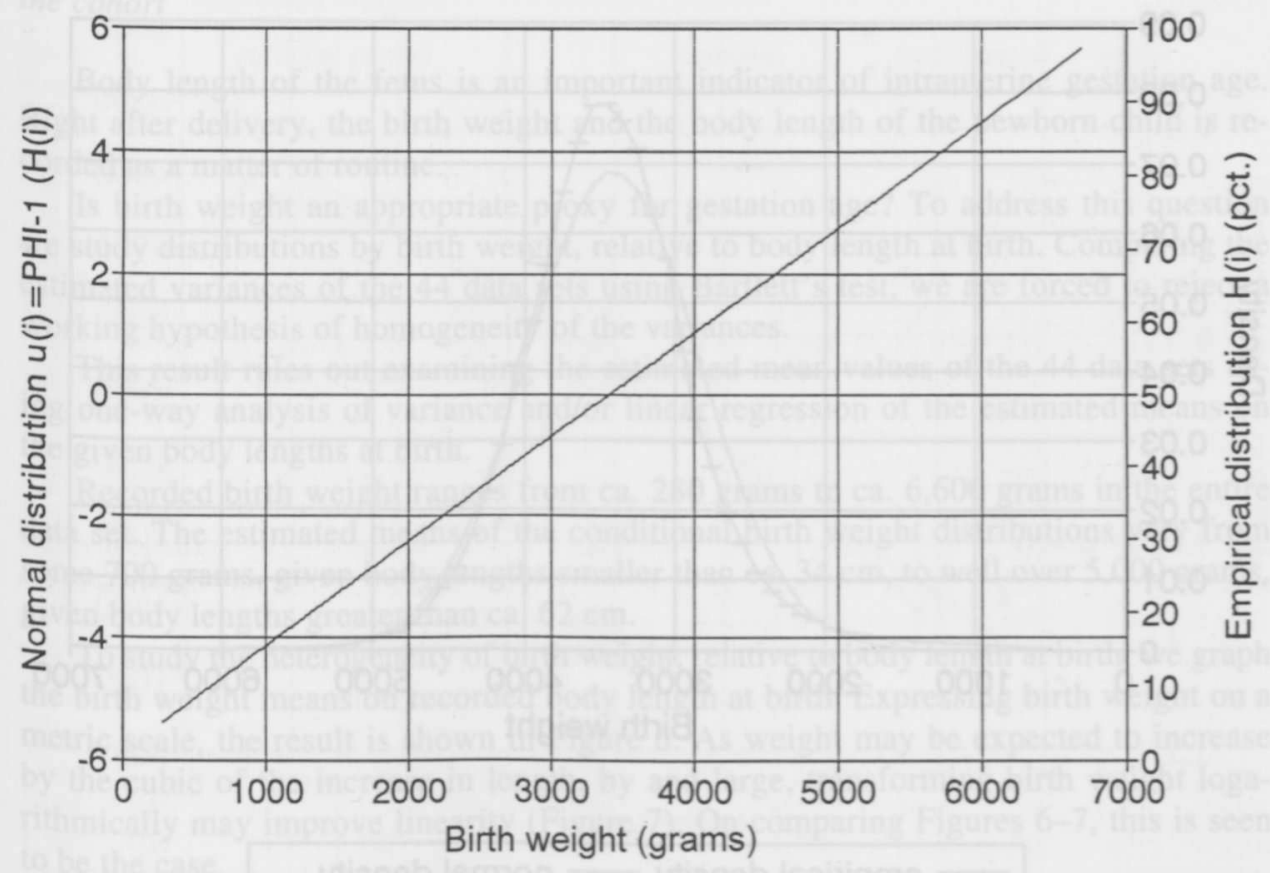

F i g u r e 3. Graphic control assumed normal distribution, height, all births.

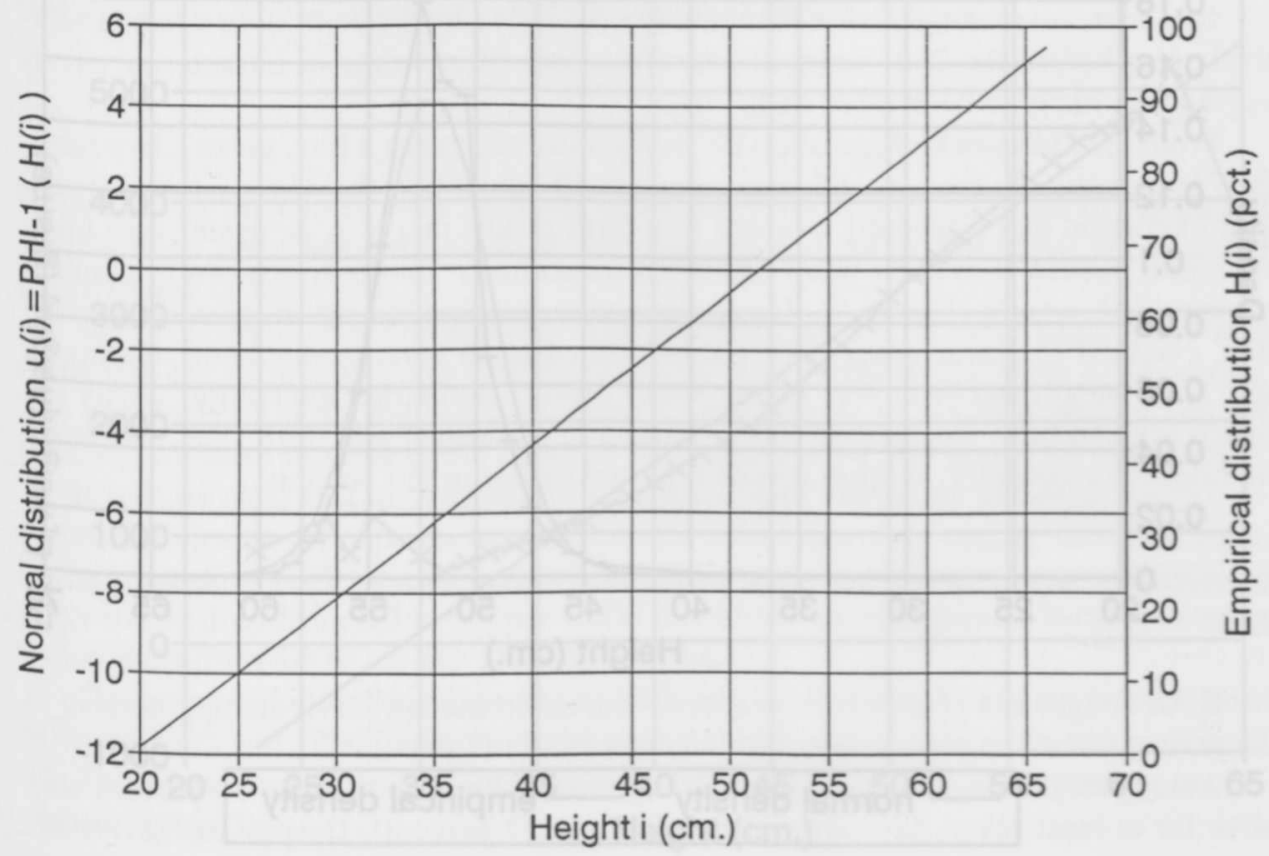


F i g u re 4. Empirical vs. normal density, all live births 1982-1990.

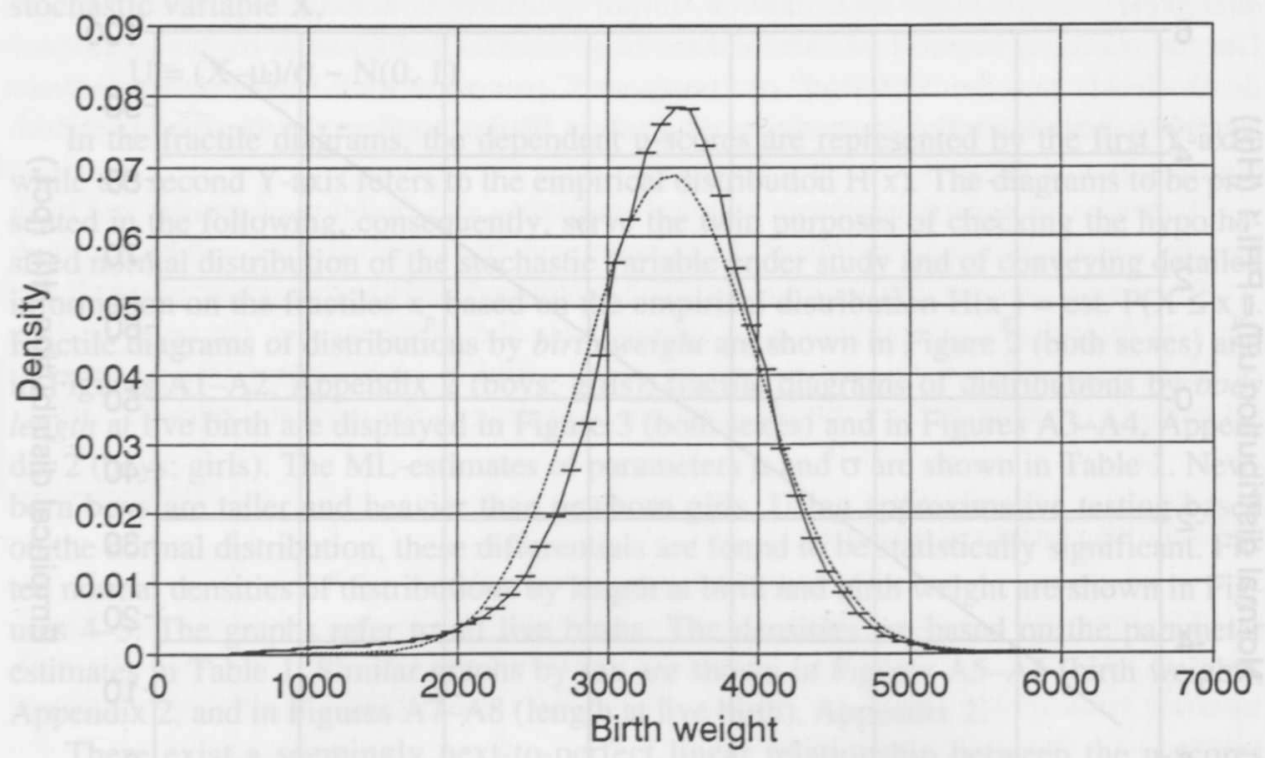

\section{empirical density - normal density}

Fi g u re 5. Empirical vs. normal density, live-born children 1982-1990.

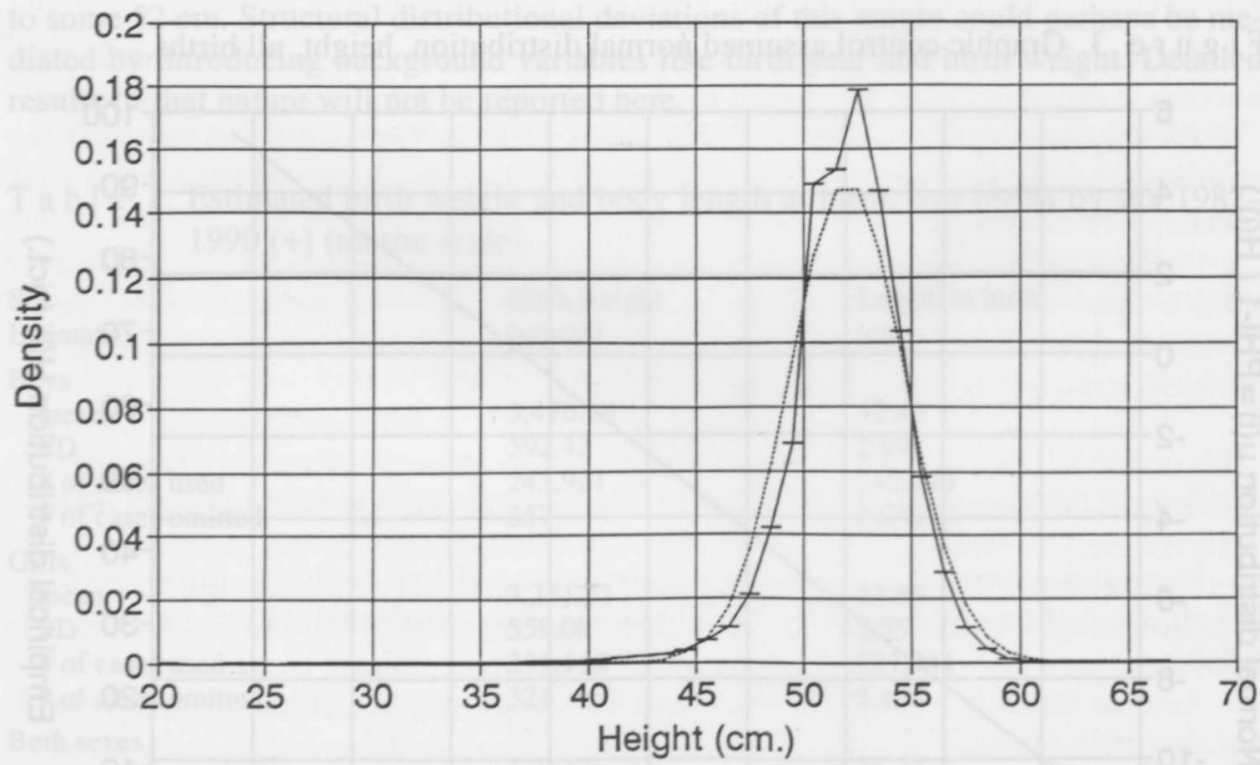


The nature of the relationship of birth weight, body length at birth, and birth year of the cohort

Body length of the fetus is an important indicator of intrauterine gestation age. Right after delivery, the birth weight and the body length of the newborn child is recorded as a matter of routine.

Is birth weight an appropriate proxy for gestation age? To address this question we study distributions by birth weight, relative to body length at birth. Comparing the estimated variances of the 44 data sets using Bartlett's test, we are forced to reject a working hypothesis of homogeneity of the variances.

This result rules out examining the estimated mean values of the 44 data sets using one-way analysis of variance and/or linear regression of the estimated means on the given body lengths at birth.

Recorded birth weight ranges from ca. 280 grams to ca. 6,600 grams in the entire data set. The estimated means of the conditional birth weight distributions vary from some 700 grams, given body lengths smaller than ca. $34 \mathrm{~cm}$, to well over 5,000 grams, given body lengths greater than ca. $62 \mathrm{~cm}$.

To study the heterogeneity of birth weight, relative to body length at birth, we graph the birth weight means on recorded body length at birth. Expressing birth weight on a metric scale, the result is shown in Figure 6. As weight may be expected to increase by the cubic of the increase in length, by and large, transforming birth weight logarithmically may improve linearity (Figure 7). On comparing Figures 6-7, this is seen to be the case.

To investigate the survivorship impact of birth weight, the latter graph is used as the basis for establishing empirically defined strategic points on the continuous weight scale. The chosen birth weight categorization is shown in Table 2. It conforms with

Fig u re 6. Plot of est. E[weight|height] against height, all live births.

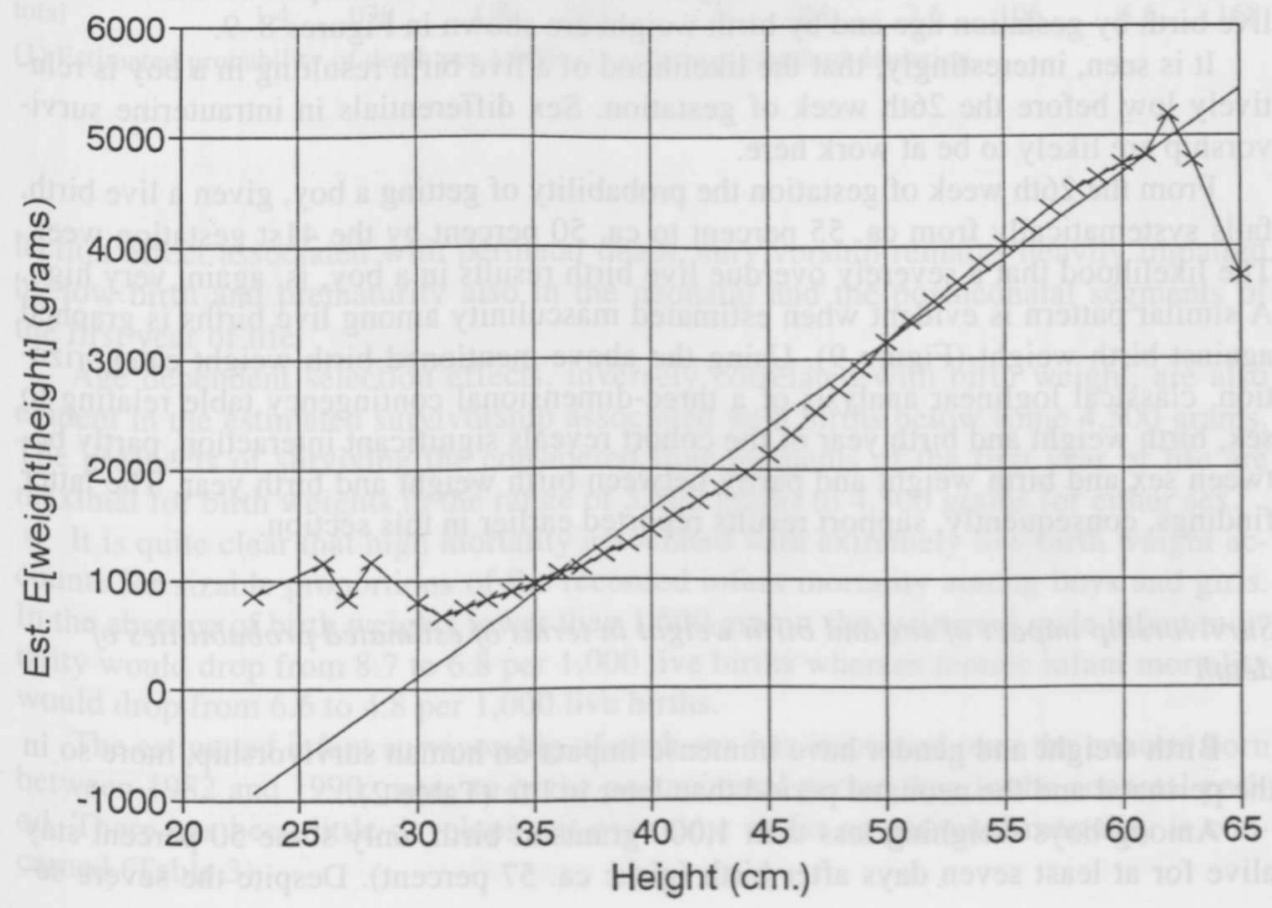


Fig u re 7. Plot of est. E[log weight|height] against height.

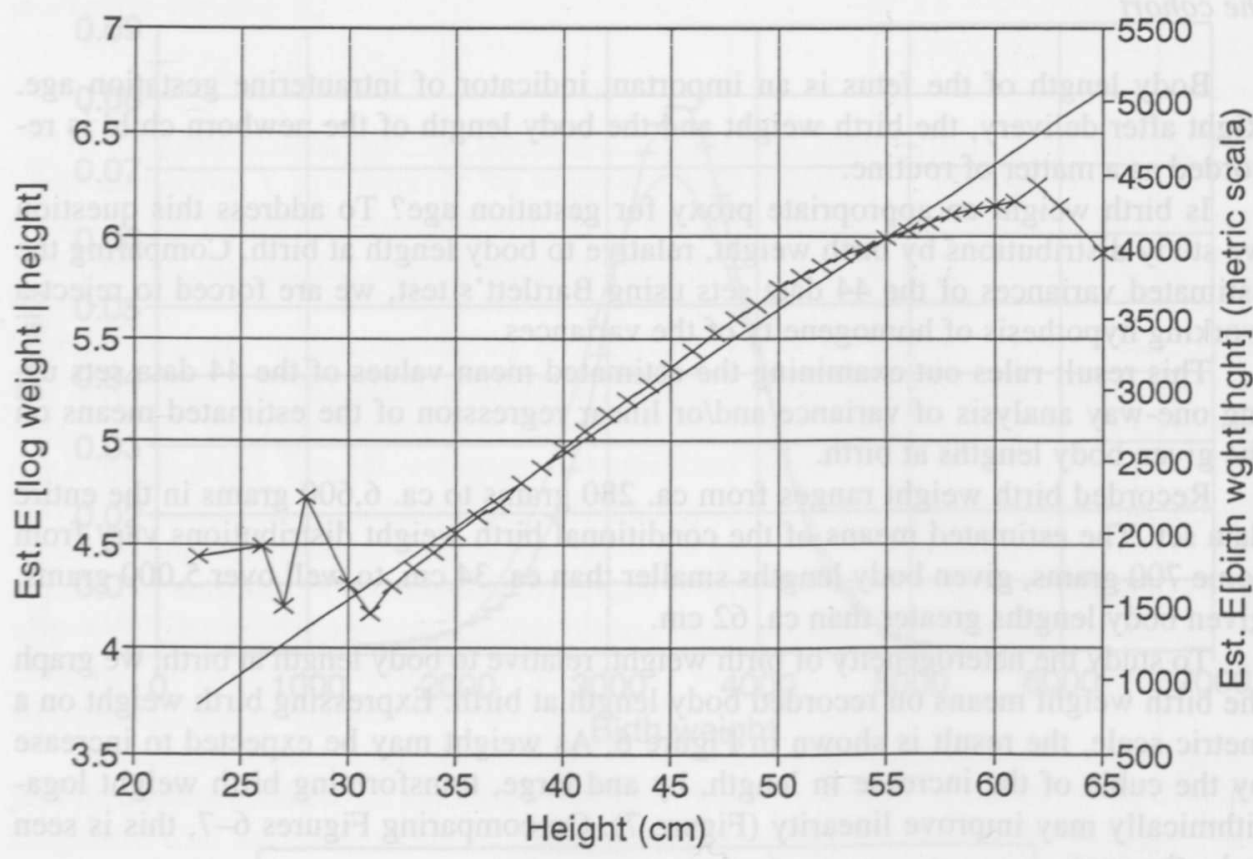

the categorization used in the annual birth weight statistics published by Danmarks Statistik since 1985. In conclusion, birth weight is a rather good proxy of gestation age represented by body length at birth in the range of ca. $35 \mathrm{~cm}$ to ca. $57 \mathrm{~cm}$.

To anticipate complex problems of interpretation related to the confounding of the impact of sex and birth weight effects in the multivariate survivorship analysis, in particular in the extremes of the birth weight distribution, estimated probabilities of male live birth by gestation age and by birth weight are shown in Figures 8-9.

It is seen, interestingly, that the likelihood of a live birth resulting in a boy is relatively low before the 26th week of gestation. Sex differentials in intrauterine survivorship are likely to be at work here.

From the 26th week of gestation the probability of getting a boy, given a live birth, falls systematically from ca. 55 percent to ca. 50 percent by the 41 st gestation week. The likelihood that a severely overdue live birth results in a boy, is, again, very high. A similar pattern is evident when estimated masculinity among live births is graphed against birth weight (Figure 9). Using the above-mentioned birth weight categorization, classical loglinear analysis of a three-dimensional contingency table relating to sex, birth weight and birth year of the cohort reveals significant interaction, partly between sex and birth weight and partly between birth weight and birth year. The latter findings, consequently, support results reported earlier in this section.

Survivorship impact of sex and birth weight in terms of estimated probabilities of death

Birth weight and gender have immense impact on human survivorship, more so in the perinatal and the neonatal period than later in life (Table 2).

Among boys weighing less than 1,000 grams at birth, only some 50 percent stay alive for at least seven days after birth (girls: ca. 57 percent). Despite the severe se- 
$\mathrm{T}$ a b l e 2. Estimated probability of death in age $[\mathrm{x}, \mathrm{y}[$ (days) (per 1,000) by gender and birth weight.

\begin{tabular}{|c|c|c|c|c|c|}
\hline \multirow{2}{*}{$\begin{array}{l}\text { Gender \& } \\
\text { birth } \\
\text { weight } \\
\text { in grams }\end{array}$} & \multicolumn{2}{|c|}{ perinatal period } & \multirow{2}{*}{$\begin{array}{l}\text { neonatal } \\
\text { period } \\
{[7,27[}\end{array}$} & \multirow{2}{*}{$\begin{array}{l}\text { postneonatal } \\
\text { period } \\
{[28,1 \text { st birth- }} \\
\text { day[ }\end{array}$} & \multirow{2}{*}{$\begin{array}{l}\text { infant } \\
\text { mortality, } \\
\text { 1st year of life }\end{array}$} \\
\hline & {$[0,1[$} & {$[1,7[$} & & & \\
\hline
\end{tabular}

(1) Estimated probability of death per 1,000; (2) estimated standard deviation

lection effect associated with perinatal death, survivorship remains heavily impaired by low birth and prematurity also in the neonatal and the postneonatal segments of the first year of life.

Age dependent selection effects, inversely correlated with birth weight, are also evident in the estimated survivorship associated with births below some 4,500 grams. The prospects of surviving the considered four segments of the first year of life are maximal for birth weights in the range of 3,500 grams to 4,500 grams for either sex.

It is quite clear that high mortality associated with extremely low birth weight accounts for sizable proportions of the recorded infant mortality among boys and girls. In the absence of birth weights lower than 1,500 grams, the estimated male infant mortality would drop from 8.7 to 6.8 per 1,000 live births whereas female infant mortality would drop from 6.6 to 4.8 per 1,000 live births.

The estimated infant survivorship of each sex has improved over the cohorts born between 1982 and 1990, more so in the postneonatal period than in the neonatal period. There has been little development over time as far as perinatal mortality is concerned (Table 3). 
Fig u re 8. Est. probability of male live birth by gestation age.

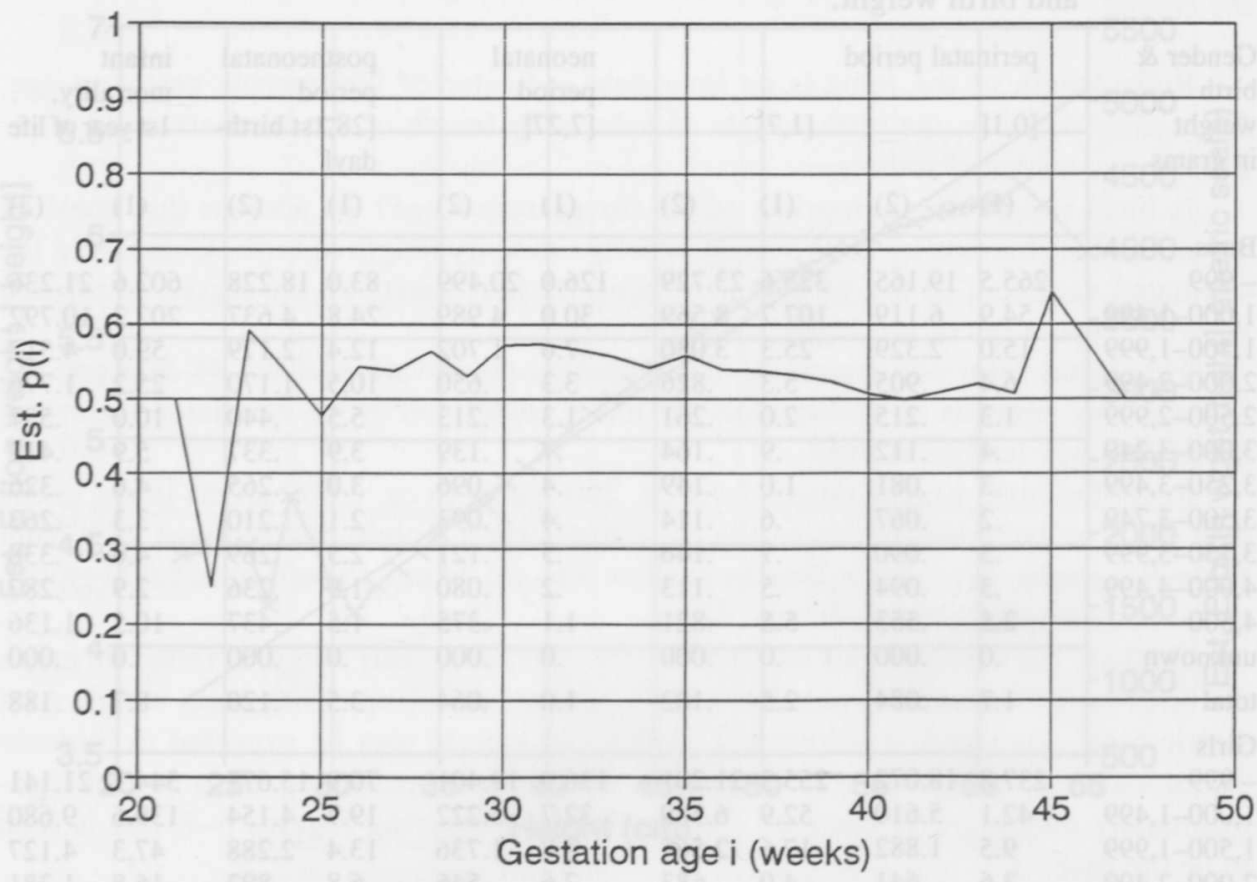

F i g u re 9. Est. probability of male live birth by birth weight.

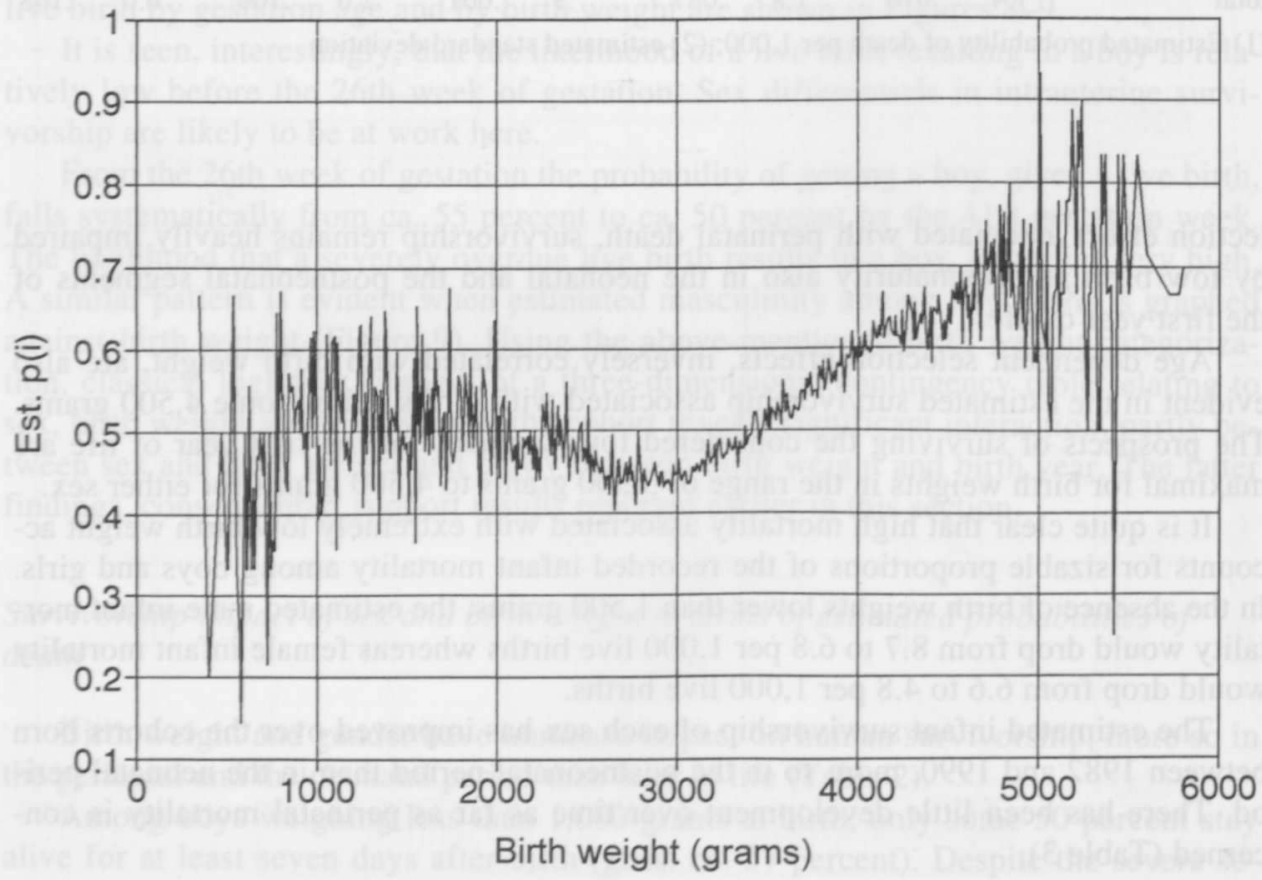


$\mathrm{T}$ a b l e 3. Estimated probability of death in age $[\mathrm{x}, \mathrm{y}[$ (per 1,000) by gender and birth year of cohort (est. standard deviation in brackets)

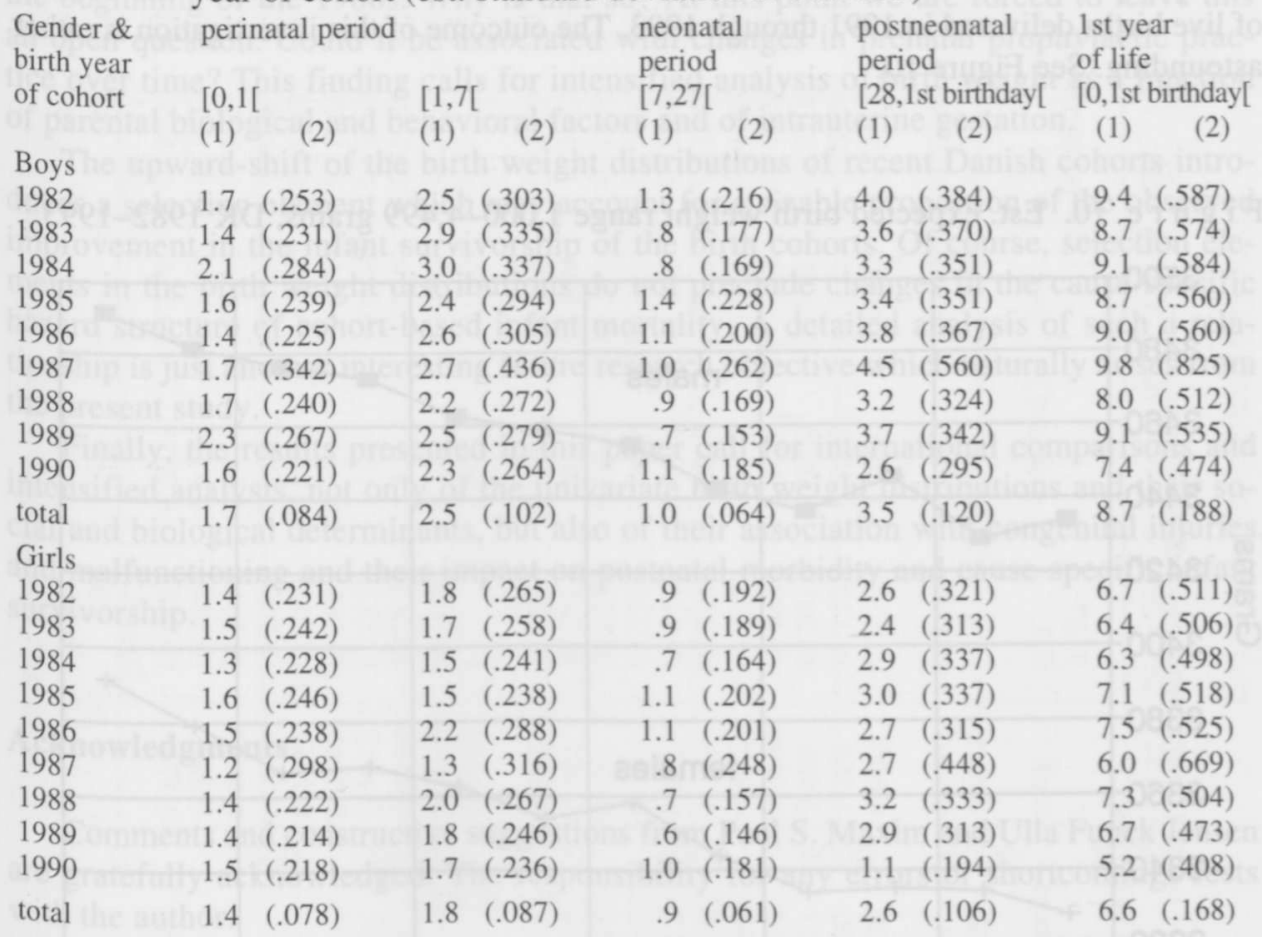

To obtain a more comprehensive impression of the anatomy of infant survivorship we performed a logistic regression of mortality on sex, birth weight and birth year of the cohort, in relation to the age of the live-born child. The results are summarized in Table 4, in Appendix 1.

The model description is not very convincing for the first 24 hours of life, and it is even poorer for the next 6 days of life. It is quite evident (Tables 2 and 4 ) that perinatal mortality is strongly associated with birth weights below about 1,500 grams, more so in the first day of life than over the next six days of life. Sex is a strongly significant explanatory variable (in favor of females) with regard to the age segments considered; the gap between the sexes widens, after the perinatal period. Interestingly, the association between perinatal mortality and the explanatory variable "birth year" is not significant. More detailed analysis is needed to disentangle the complexity of the relationship of the considered explanatory variables with regard to perinatal mortality.

Proceeding to the neonatal and postneonatal age segments we see that age-specific probability of death may now be described by logistic regression models incorporating sex, birth weight and birth year as explanatory variables. The birth weight factor is stronger and the birth year factor weaker in the neonatal age segment relative to the postneonatal period. The differences across the neonatal and the postneonatal age segments are very significant statistically (the test results supporting this finding will not be reported here).

As infant survivorship by sex has been found to be strongly associated with birth weight (Tables 2 and 4) and birth year of cohort (Tables 3 and 4), it might be worth examining whether cohort-specific birth weight would be differential by birth year of the cohort. To study this relationship we plotted the estimated cohort-specific birth 
weight in the range $1,000-4,499$ grams on the birth year of the cohort. To extend the time perspective we included estimates based on published statistics for the cohorts of live births delivered in 1991 through 1993. The outcome of this investigation is rather astounding. See Figure 10.

F i g u r e 10. Est. expected birth weight range 1,000-4,499 grams, DK 1982-1993.

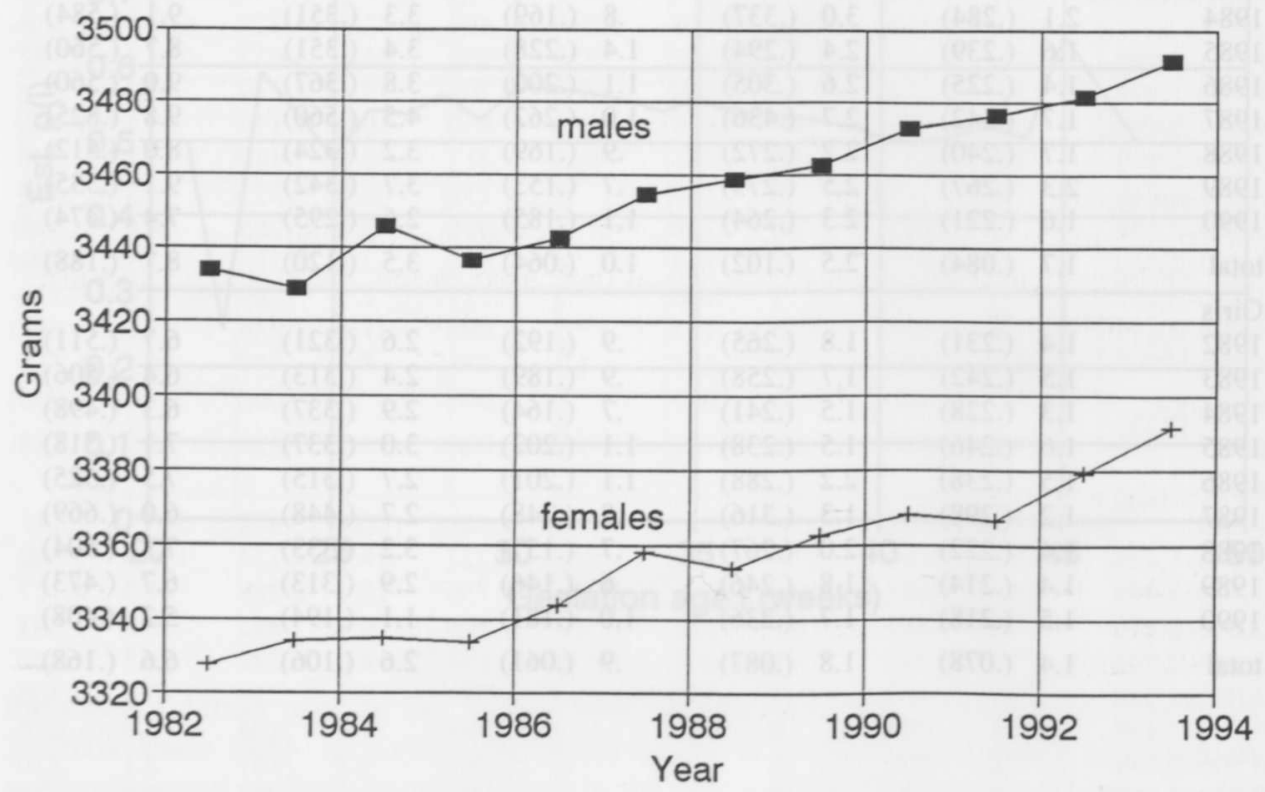

Over the period 1982 to $1993 / 94$ the estimated expected birth weights in the range 1,000 to 4,499 grams have increased by some 50 grams for each of the sexes almost linearly with time. In consequence of the findings summarized above, a further decrease in Danish cohort-based infant mortality is to be expected also in the beginning of the 1990s and this is exactly what we see. According to measurements reported by Danmarks Statistik $(1995,69)$, the cohort-based infant mortality of the boys born in 1992 amounted to 7.2 per 1,000 live births (girls: 5.5 per 1,000 live births). This brings Denmark to the same level with the other Nordic countries as far as infant survivorship is concerned.

\section{Conclusion}

In this paper we have made a first approach to the study of recent Danish infant survivorship as a function of biosocial factors related to the parents and to intrauterine gestation; the focus of the paper having been on infant survivorship as a function of the sex, birth weight and birth year of the cohorts.

The paper corroborates established knowledge by singling out birth weight as a very influential intermediate determinant of infant survivorship. Bringing fetuses with very low birth weight alive into this world may severely impair infant survivorship and add negatively to the shaping of the future social life and the career opportunities of the newborn child. 
The estimated expected birth weight has increased almost linearly with time since the beginning of the $1980 \mathrm{~s}$. Why is that so? At this point we are forced to leave this an open question. Could it be associated with changes in prenatal prophylactic practice over time? This finding calls for intensified analysis of birth weight as a function of parental biological and behavioral factors and of intrauterine gestation.

The upward-shift of the birth weight distributions of recent Danish cohorts introduces a selection element which may account for a sizable proportion of the observed improvement in the infant survivorship of the birth cohorts. Of course, selection elements in the birth weight distributions do not preclude changes in the cause-specific hazard structure of cohort-based infant mortality. A detailed analysis of such a relationship is just another interesting future research objective which naturally arises from the present study.

Finally, the results presented in this paper call for international comparisons and intensified analysis, not only of the univariate birth weight distributions and their social and biological determinants, but also of their association with congenital injuries and malfunctioning and their impact on postnatal morbidity and cause-specific infant survivorship.

\section{Acknowledgments}

Comments and constructive suggestions from Paul S. Maxim and Ulla Funck Jensen are gratefully acknowledged. The responsibility for any errors or shortcomings rests with the author.

\section{References}

Belsey, M. 1993. Child health and development: health of the newborn. In: Birth Risks, edited by Baum, J. David. (Nestle Nutrition Workshop Series Vol. 31, 1-24). New York: Raven Press.

Bouvier, L.F. and J. van der Tak 1976. Infant mortality - progress and problems. Population Bulletin 31(1):1-32.

Cardozo, L.D., D.M. Gibb, J.W. Studd and D.J.Cooper.1982. Social and obstetric features associated with smoking in pregnancy. British Journal of Obstetrics and Gynaecology 89(8):622-7.

Cederqvist, L.L., G. Eddey; N. Abdel Latif and S.D. Litwin. 1984. The effect of smoking during pregnancy on cord blood and maternal serum immunoglobulin levels. American Journal of Obstetrics and Gynaecology 148(8):1123-6.

Danmarks Statistik. 1995. Befolkningens bevægelser (Movements of the population). Copenhagen: Danmarks Statistik.

Hansen, H.O. (1993). Elementær demografi. Med statistikprogram DEMOPACK og data på diskette. (Elemental demography. With statistics package DEMOPACK and data on diskette). Copenhagen: Akademisk Forlag.

Hansen, H.O. 1994. A User's Guide to MINISTAT. University of Copenhagen, Institute of Statistics. (unpublished).

Phelan, J.P. 1980. Diminished fetal reactivity with smoking. American Journal of Obstetrics and Gynaecology $15,136(2): 230-3$.

Tambyraja, R.L.and S.S. Ratnam. 1982. The small fetus: growth retarded and preterm. Clinics in Obstetrics and Gynaecology 9(3):517-37. 


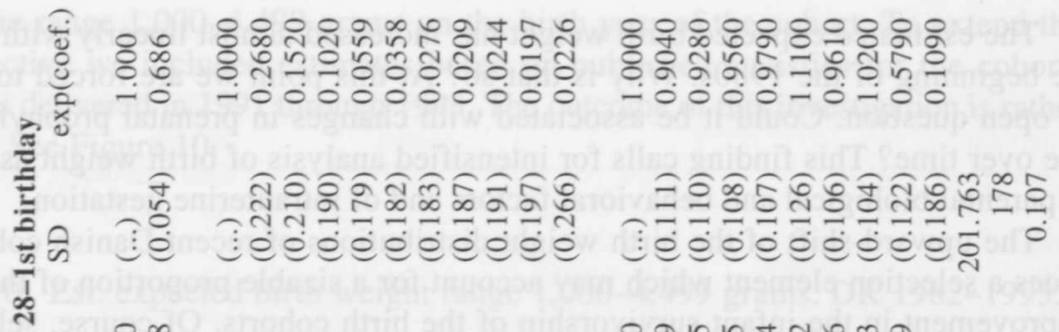

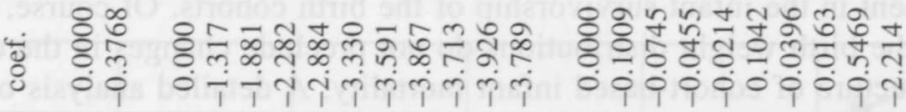

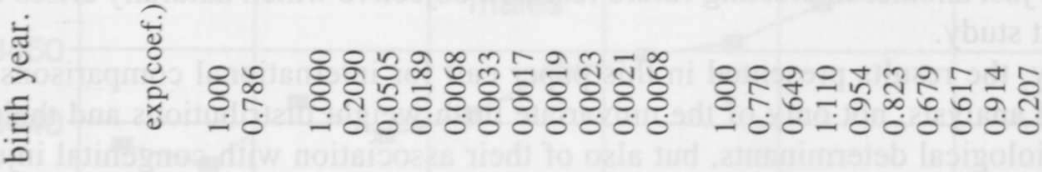

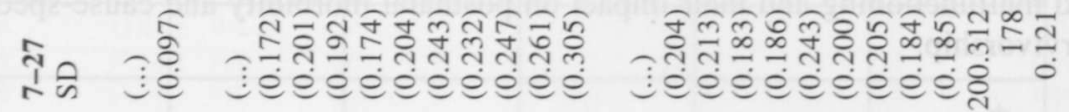

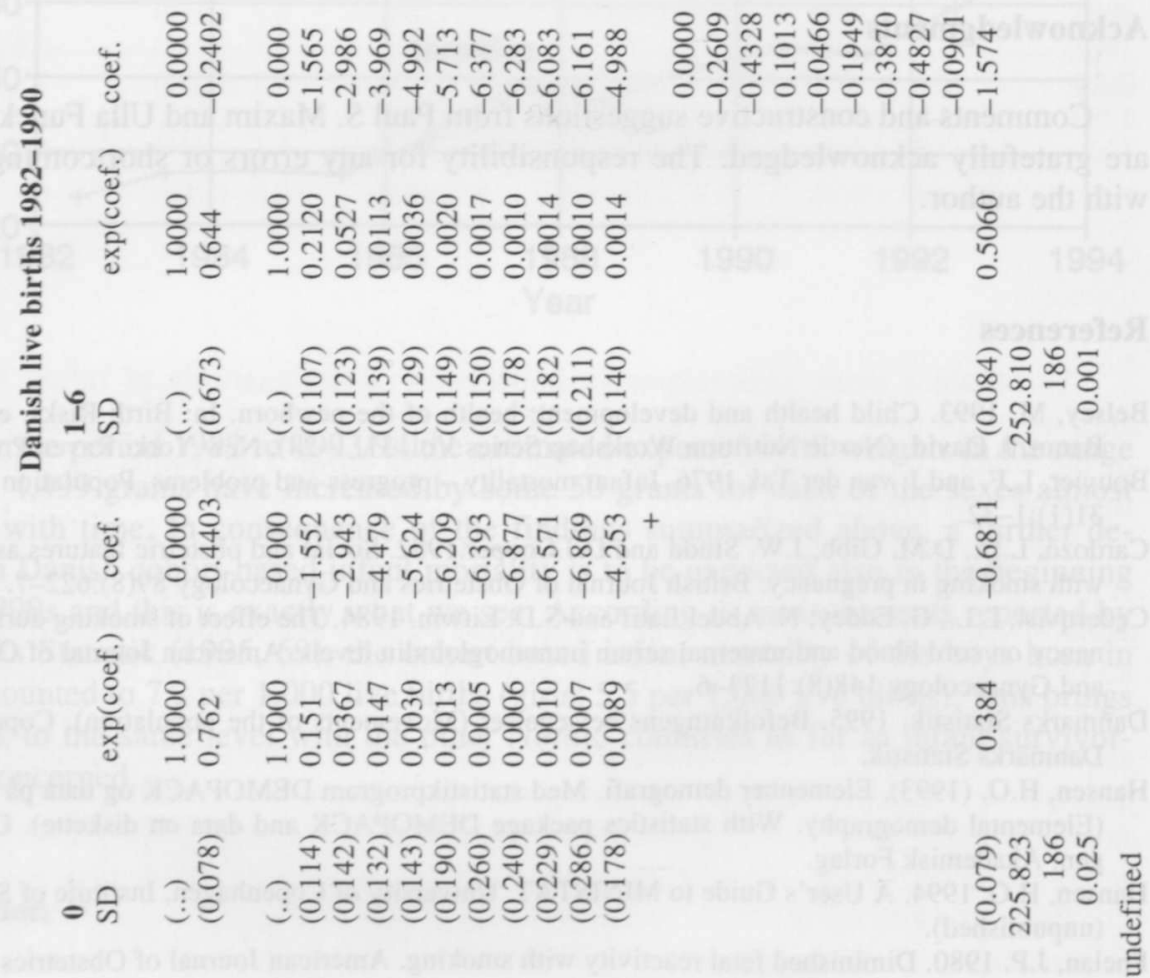

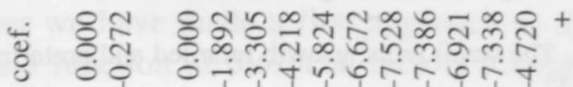

๙ั

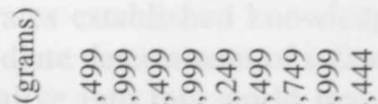

औ. 
Appendix 2.

Fi g u re A1. Fractile diagram, birth weight, all boys.

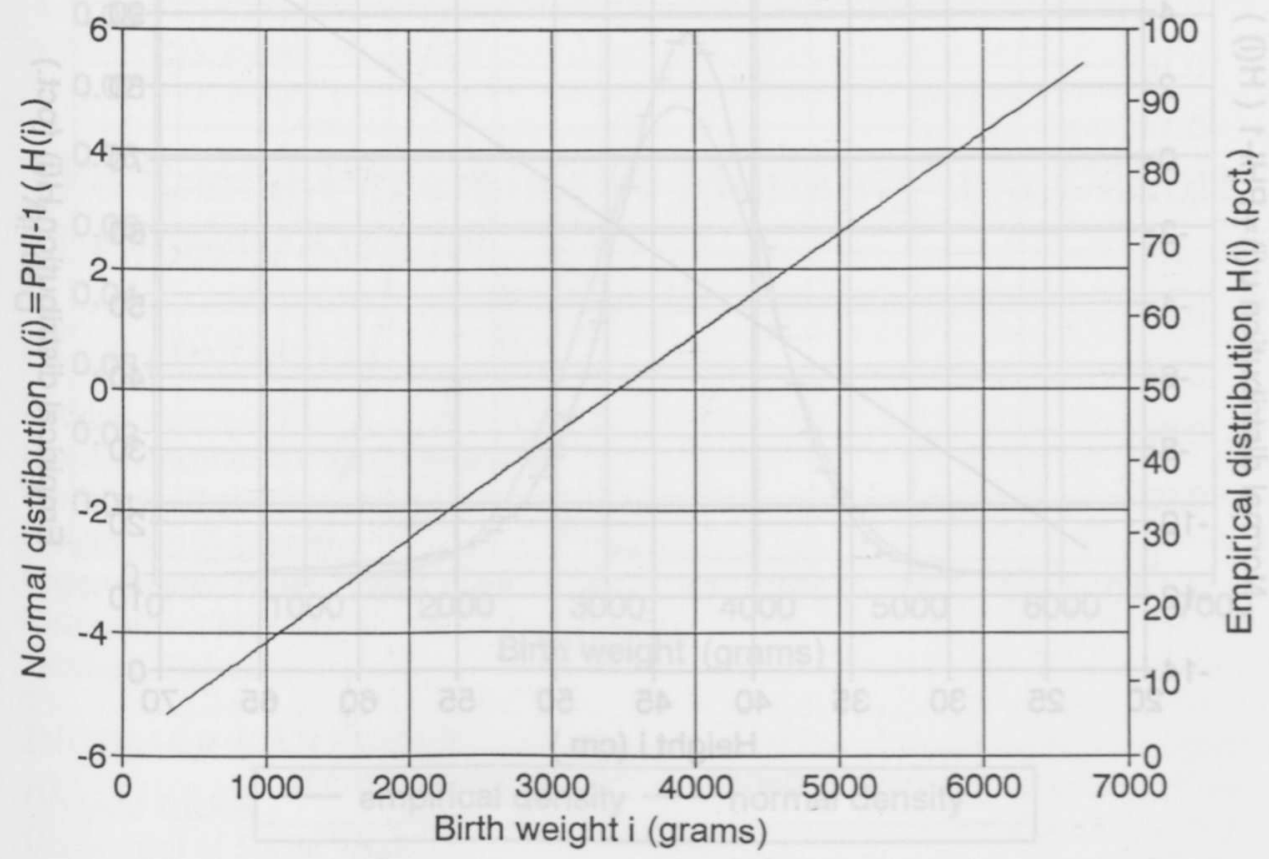

Fig u r e A2. Fractile diagram, birth weight, all girls.

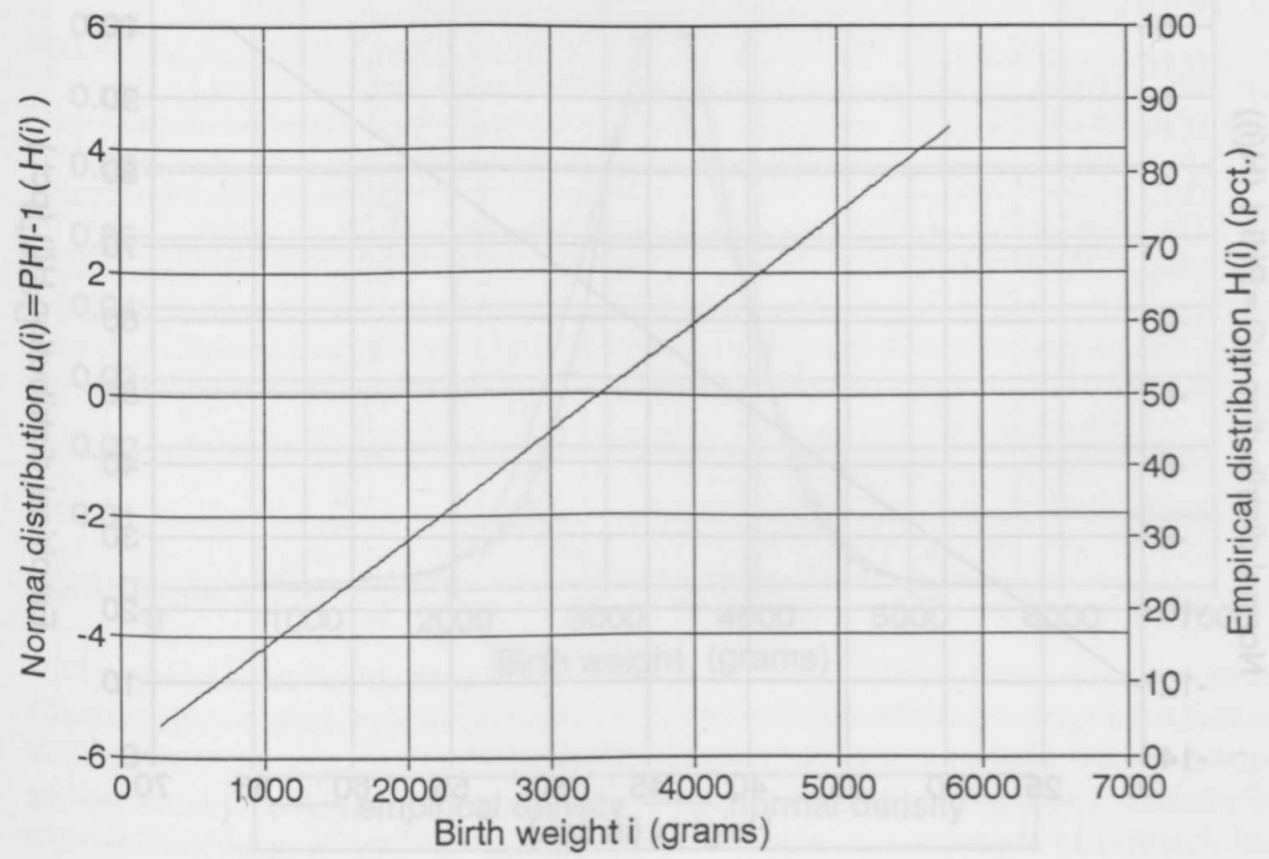


Fig u re A3. Fractile diagram, height at live birth, all boys.

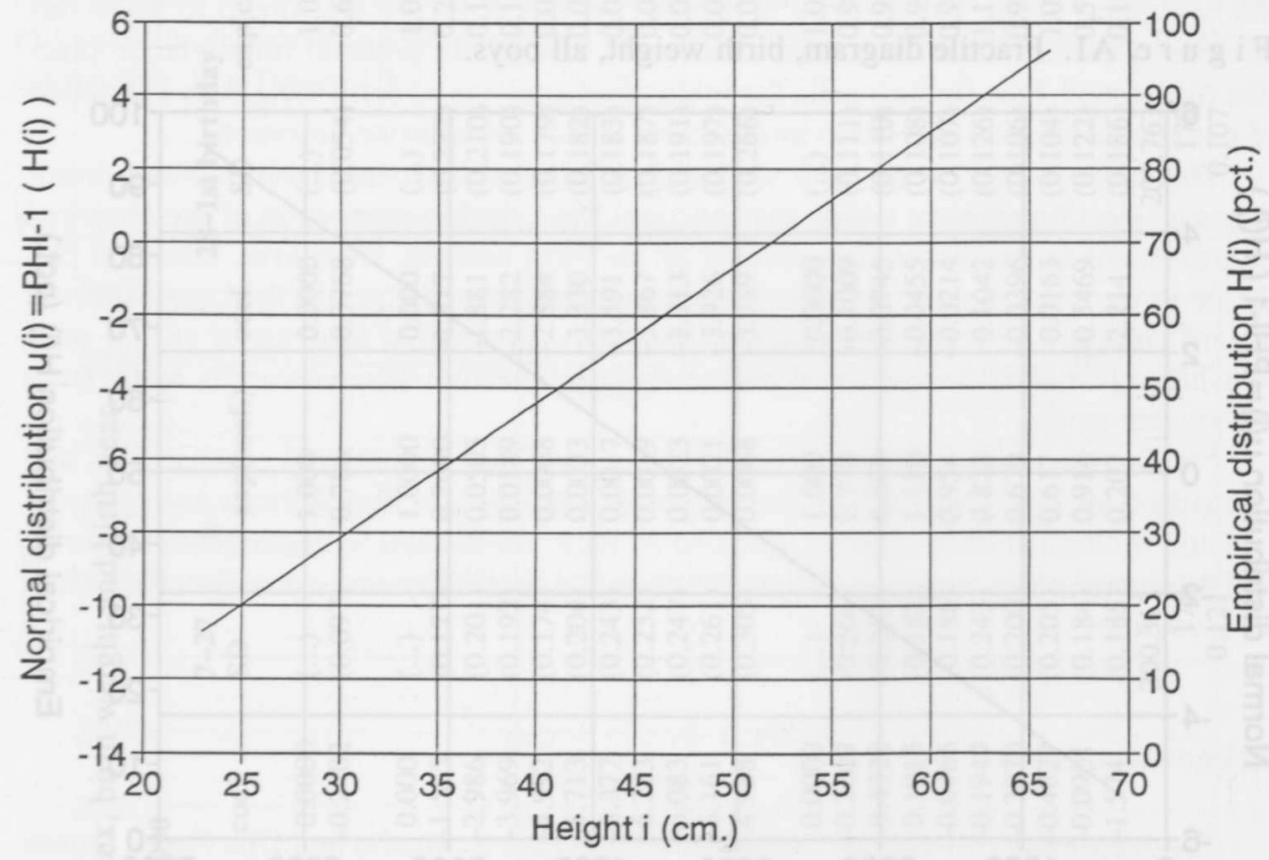

Fi g u r e A4. Fractile diagram, height at live birth, all girls.

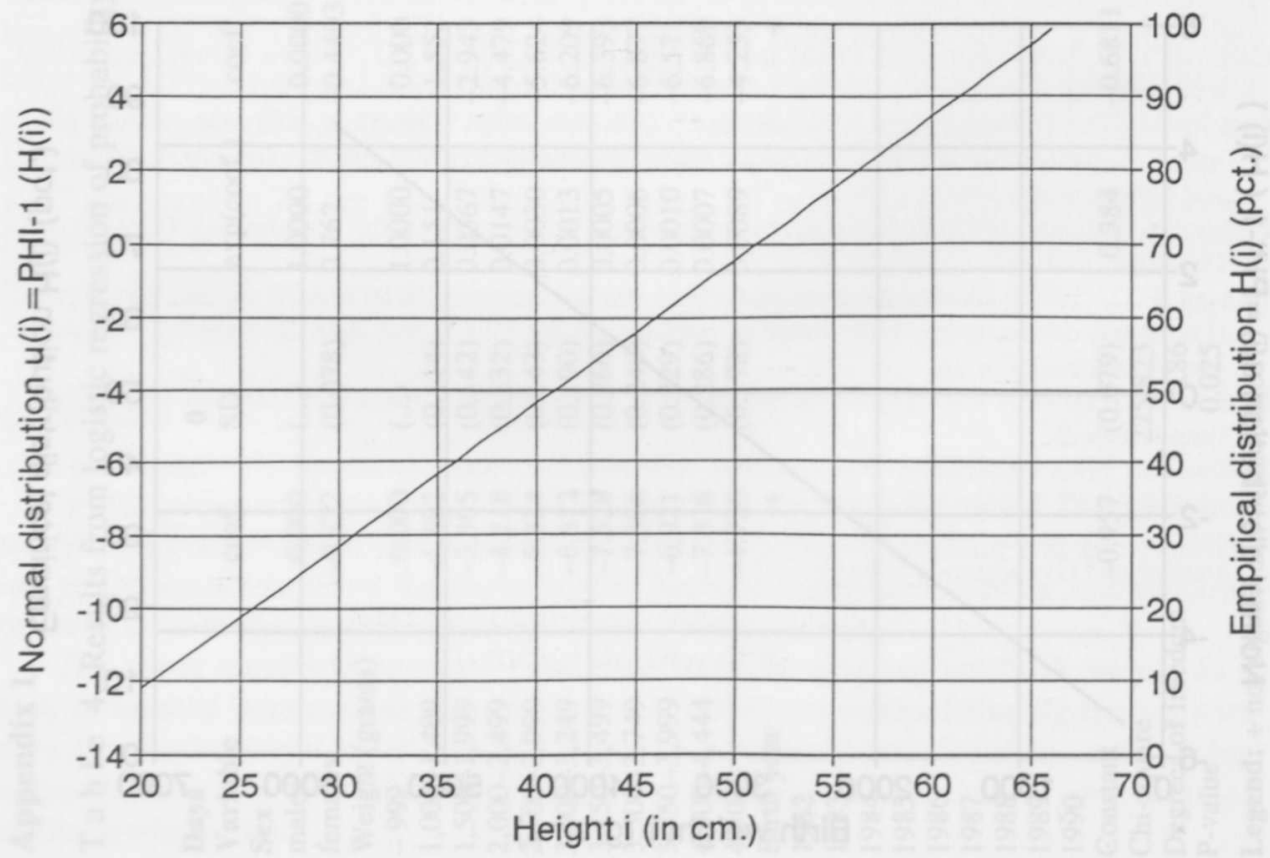


Fi g u re A5. Empirical vs. normal density, boys born 1982-1990.

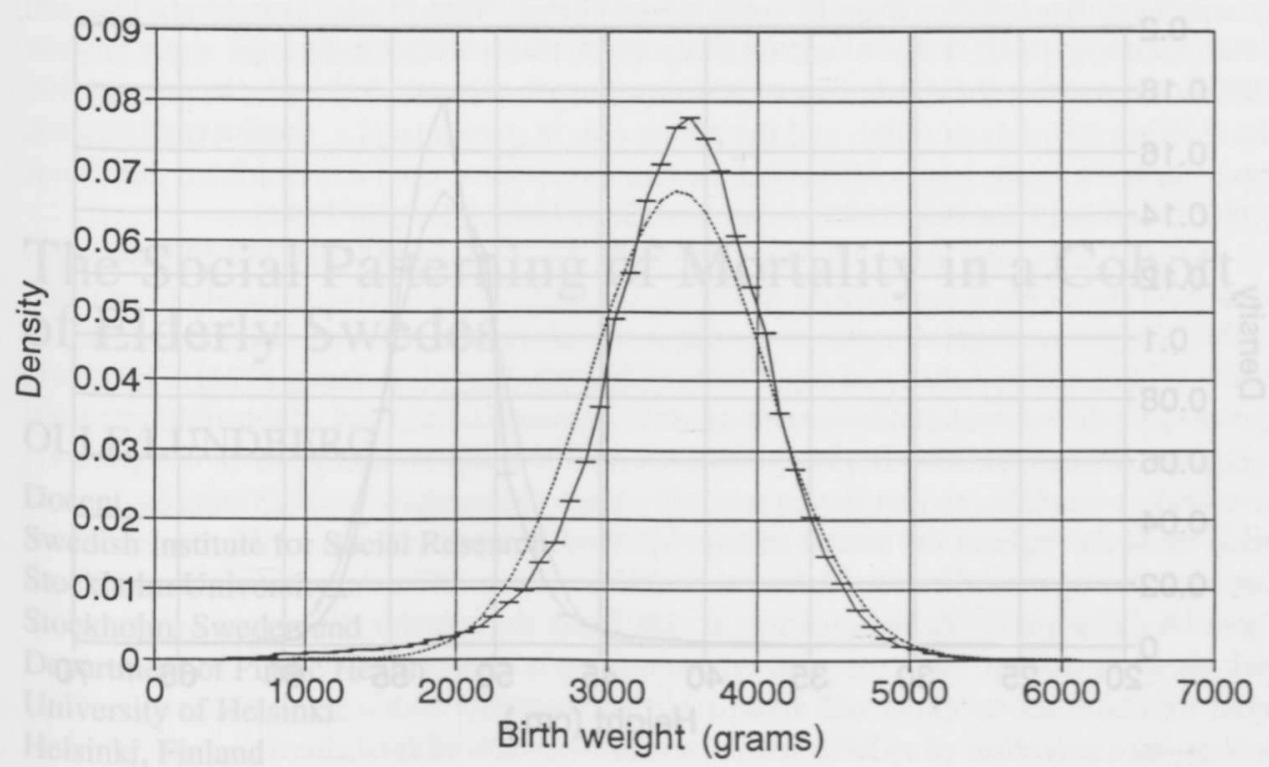

empirical density - normal density

Fi g u r e A6. Empirical vs. normal density, girls born 1982-1990.

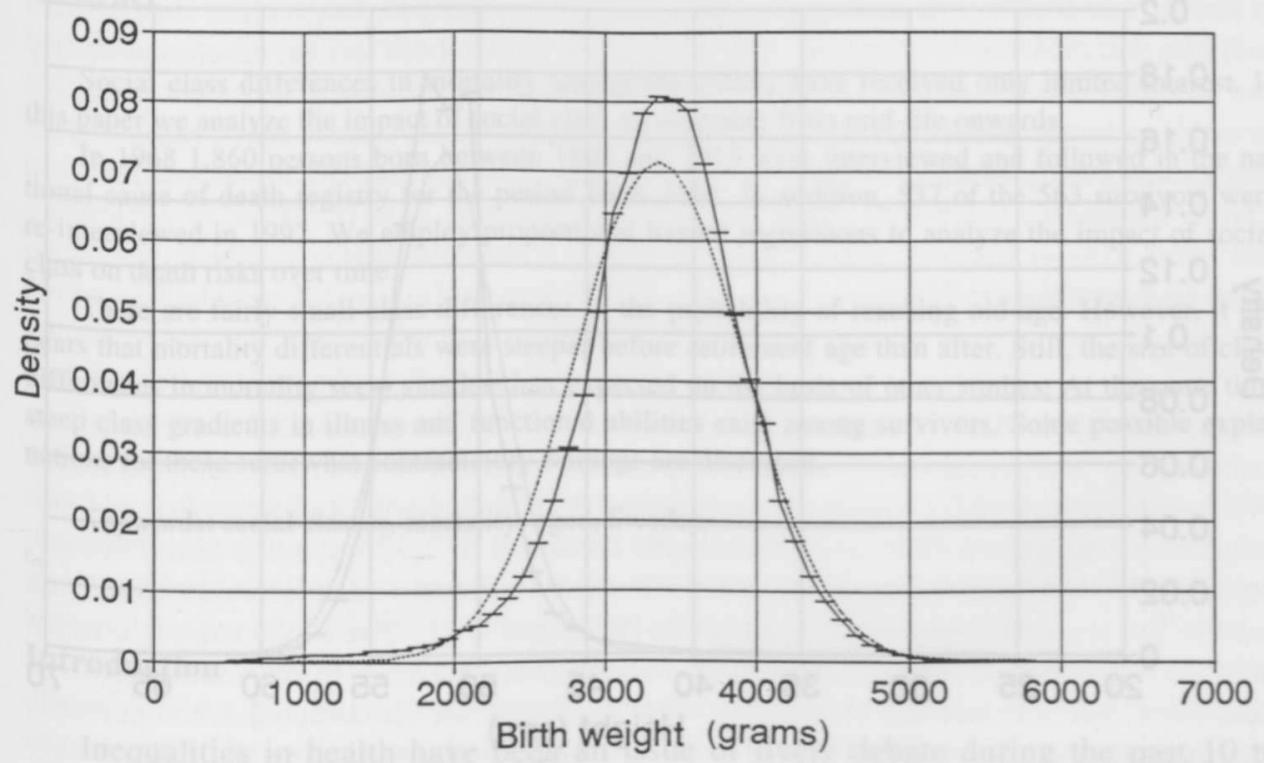


Fi g u re A7. Empirical vs. normal density, boys born 1982-1990.

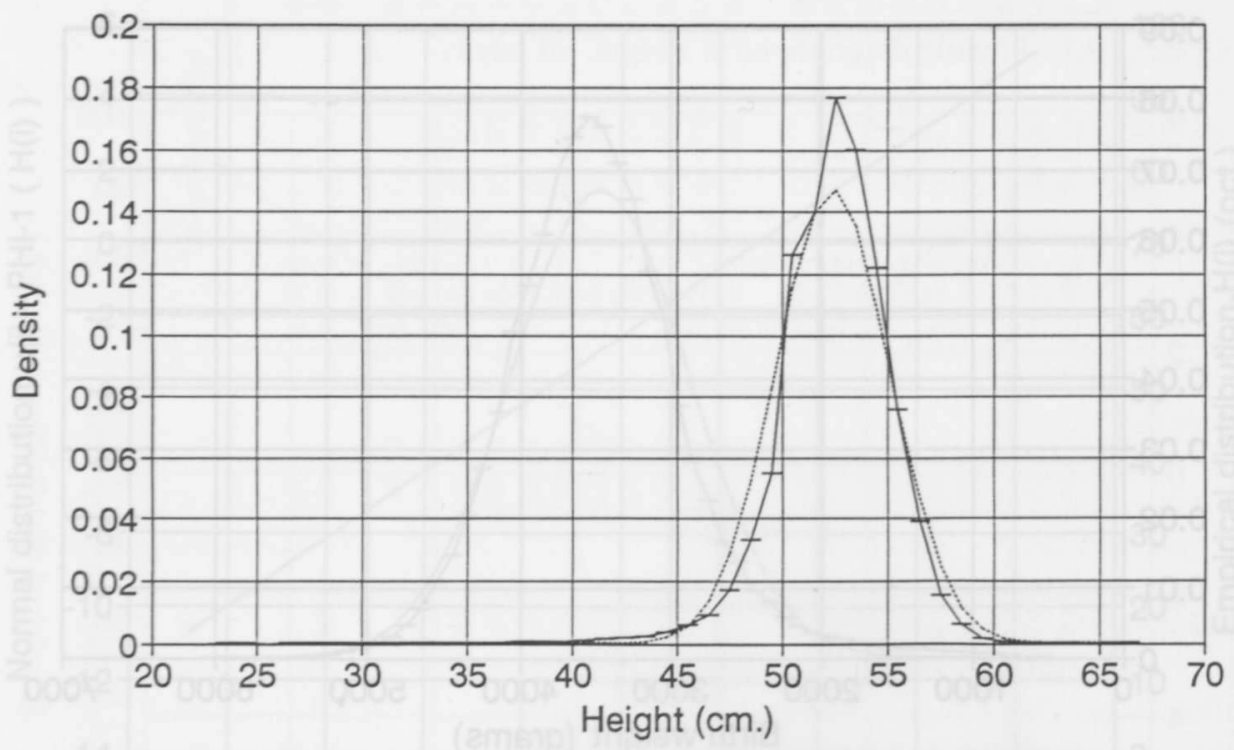

Fig u re A8. Empirical vs. normal density, girls born 1982-1990.

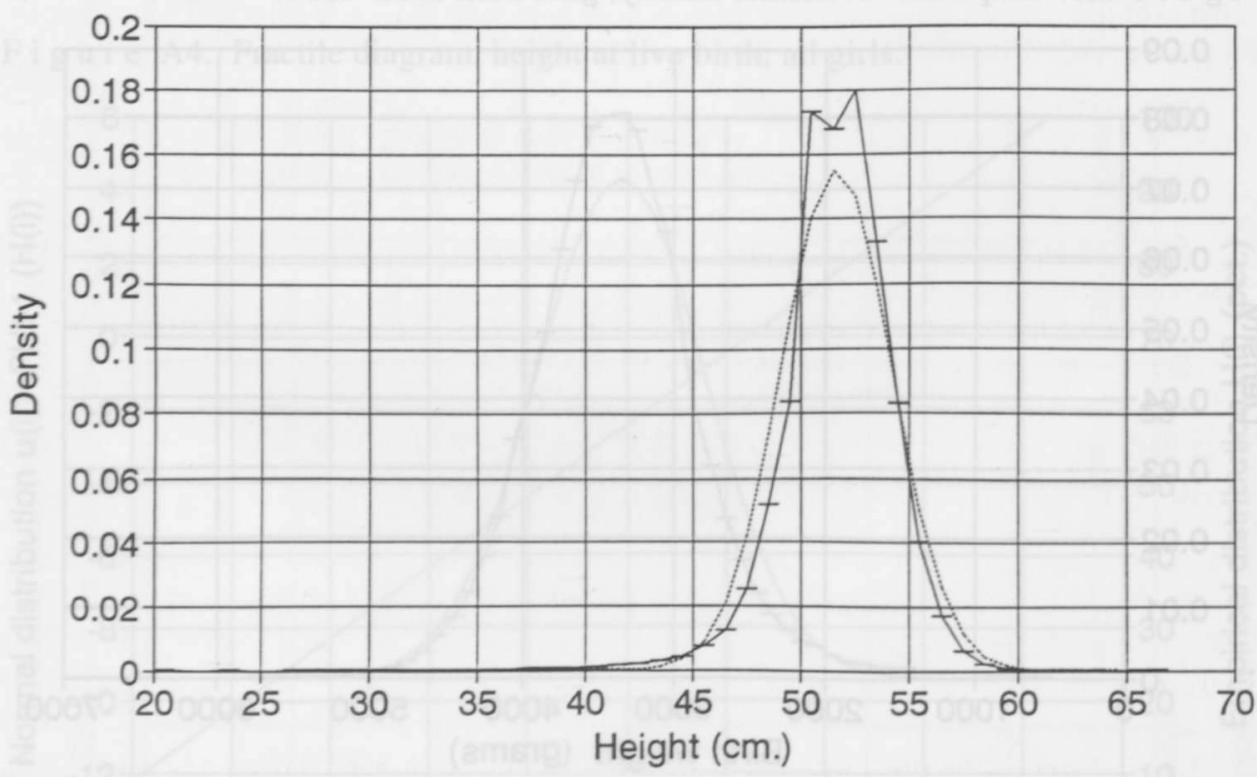

